\title{
ON SANDWICH THEOREMS FOR CERTAIN SUBCLASSES OF ANALYTIC FUNCTIONS INVOLVING A LINEAR OPERATOR
}

\author{
T. N. Shanmugam, S. Sivasubramanian And S. OWA
}

Abstract. The purpose of this present paper is to derive some subordination and superordination results for certain normalized analytic functions involving complex order in the open unit disk, acted upon by Carlson-Shaffer operator. Relations of the results, which are obtained in this paper, with various known results are also presented.

Mathematics subject classification (2000): 30C80, 30C45.

Key words and phrases: differential subordinations, differential superordinations, dominant, subordinant.

\section{REFERENCES}

[1] R. M. Ali, V. Ravichandran, M. Hussain Khan and K. G. Subramanian, Differential sandwich theorems for certain analytic functions, Far East J.Math. Sci. (FJMS), (FJMS), 15, (1) (2005), 87-94.

[2] T. BULBOACĂ, A class of superordination-preserving integral operators, Indag. Math., New Ser., 13, (3) (2002), 301-311.

[3] T. BulbOACĂ, Classes of first-order superordinations, Demonstr. Math., 35, (2) (2002), $287-292$.

[4] B. C. CARLSON, D. B. SHAFFER, Starlike and prestarlike hypergeometric functions, SIAM J. Math. Anal., 15, (1984), 737-745.

[5] S. S. Miller, P. T. Mocanu, On some classes of first order differential subordinations, The Michigan Matematical Journal, 32, (1985), 185-195.

[6] S. S. Miller, P. T. Mocanu, Differential Subordinations: Theory and Applications, Pure and Applied Mathematics No. 225, Marcel Dekker, New York, (2000).

[7] S. S. Miller, P. T. Mocanu, Subordinants of differential superordinations, Complex Variables, 48 , (10) (2003), 815-826.

[8] M. Obradović, S. Owa, On certain properties for some classes of starlike functions, J. Math. Anal. Appl., 145, (2) (1990), 357-364.

[9] G. I. OROS, On a class of holomorphic functions defined by Salagean differential operator, Complex Var. Theory Appl., 50, (4) (2005), 257-264.

[10] S. RuscheweYh, New criteria for univalent functions, Proc. Amer. Math. Soc., 49, (1975), $109-115$.

[11] T. N. Shanmugam, V. Ravichandran and S. Sivasubramanian, Differential Sandwich Theorems for some subclasses of Analytic Functions, Austral. Math. Anal. Appl., 3, (1) Article 8, (2006), pp. 11.

[12] H. M. SRIVAStaVA, A. Y. LASHIN, Some applications of the Briot-Bouquet differential subordination, JIPAM. J. Inequal. Pure Appl. Math., 6, (2) (2005), Article 41, 7 pp.

[13] N. TUNESKI, On certain sufficient conditions for starlikeness, Internat J. Math. Math. Sci., 23, (8) 2000, 521-527. 